\title{
Research on Multi-Component Gas Optical Detection System Based on Conjugated Interferometer
}

\author{
Xin GUI ${ }^{*}$, Yuheng TONG ${ }^{2}$, Honghai $\mathrm{WANG}^{1}$, Haihu $\mathrm{YU}^{1}$, and Zhengying $\mathrm{LI}^{1,2}$ \\ ${ }^{1}$ National Engineering Laboratory for Fiber Optic Sensing Technology, Wuhan University of Technology, Wuhan, \\ 430070, China \\ ${ }^{2}$ Key Laboratory of Fiber Optic Sensing Technology and Information Processing, Ministry of Education, Wuhan \\ University of Technology, Wuhan, 430070, China \\ ${ }^{*}$ Corresponding author: Xin GUI E-mail: guixin@whut.edu.cn
}

\begin{abstract}
An optical multi-component gas detection system based on the conjugated interferometer (CI) is proposed and experimentally demonstrated. It can realize the concentration detection of mixture gas in the environment. The CI can transform the absorption spectrum of the target gases to a conjugated emission spectrum, when combining the CI with the broadband light source, the spectrum of output light matches well with the absorption spectrum of target gases. The CI design for different target gases can be achieved by replacing the kind of target absorbing gas in the CI filter. The traditional fiber gas sensor system requires multiple light sources for detection when there are several kinds of gases, and this problem has been solved by using the CI filter combined with the broadband light source. The experimental results show that the system can detect the concentration of multi-component gases, which are mixed with $\mathrm{C}_{2} \mathrm{H}_{2}$ and $\mathrm{NH}_{3}$. Experimental results also show a good concentration sensing linearity.
\end{abstract}

Keywords: Gas conjugated interference filter; gas sensing; spectral absorption

Citation: Xin GUI, Yuheng TONG, Honghai WANG, Haihu YU, and Zhengying LI, "Research on Multi-Component Gas Optical Detection System Based on Conjugated Interferometer," Photonic Sensors, 2017, 7(3): 261-269.

\section{Introduction}

The multi-component gas detection has played a significant role in many fields, such as in the atmospheric condition detection, the safe operation of the transformer, industrial process control, and medical diagnosis [1-3]. The optical gas detection technology based on Beer-Lambert's law is widely applied in detecting different kind of gases such as carbon monoxide, carbon dioxide, methane, hydrogen, and acetylene. Tunable diode laser absorption spectroscopy (TDLAS) is one of the most commonly used gas detection methods with high sensitivity. The system based on the distributed feedback (DFB) laser [4-5] has narrow line-widths whose wavelength can be precisely tuned to match a gas absorption line, giving high spectral power density. However, DFB lasers have several disadvantages, for example, they are generally expensive and gas specific. Hence, a separate DFB laser is often required for each gas absorption line of interest. Besides, it cannot be applied in multiple-component gas due to the wide distribution of gas absorption lines.

Received: 13 February 2017 / Revised: 27 April 2017

(C) The Author(s) 2017. This article is published with open access at Springerlink.com

DOI: $10.1007 / \mathrm{s} 13320-017-0405-5$

Article type: Regular 
The selection of a light source plays a vitally important role in the spectrum absorption gas sensor system. Worldwide researchers have proposed various methods to solve this problem. The bandwidth of a broadband source is typically $20 \mathrm{~nm}$ $-100 \mathrm{~nm}$ and can cover absorption lines of multiple types of gases, such as $\mathrm{C}_{2} \mathrm{H}_{2}, \mathrm{CO}, \mathrm{CO}_{2}, \mathrm{H}_{2} \mathrm{~S}$, and $\mathrm{NH}_{3}$, which have absorption lines in the range of $1510 \mathrm{~nm}$ to $1590 \mathrm{~nm}$. For its stable performance, long service life, easy operation, and wide range, the broadband source is widely used in the gas detection. Broadband sources combined with optical filters to choose the gas absorption lines provide a good choose for the gas detection system. Applications of a broadband light source with filters of the gas sensor have been reported of which some are based on the fiber Bragg grating (FBG) [6], Fabry-Perot etalon [7-9], and the filter plate [10, 11]. In addition, using the method of the comb filter [12], the broadband light source can accurately match a plurality of $\mathrm{CH}_{4}$ gas absorption lines. Fiber interferometers have been reported using as a filter, for example, the filters based on the Michelson interferometer principle [12] and Mach-Zehnder interference [13] principle combining with the FBG or other optical devices to achieve quality narrowband transmission filter can be used in the vicinity of the gas absorption $1550 \mathrm{~nm}$. Spectral matching with the gas absorption lines is the most challenging part of the filter approach.

Recently, we have proposed single gas detection method based on the conjugated interferometer (CI) filter [14], which transforms the absorption spectrum of the sample gas to a conjugated emission spectrum. Here, a comb light source which emits light at gas absorption lines is produced. However, for the previous case, the CI filter essentially acts as a single gas. In the present study, based on the broadband light source, the CI filter can be designed as the output of the wavelength light source matching the absorption lines of the multi-target gases so as to be used in the multi-gases detection system. By changing the gases in the CI filter, it can produce the optical output corresponding to multiple gases, and the output exactly corresponds to their intrinsic absorption lines of the gases, which greatly improves the low sensibility caused by the broadband light source. It provides a solution to the simplicity of gas selection in the light source. In the paper, the experimental results show that the system can detect multi-component gases concentrations, which are mixed with $\mathrm{C}_{2} \mathrm{H}_{2}$ and $\mathrm{NH}_{3}$, and achieve a good concentration sensing linearity.

\section{Principle of conjugated interferometer}

\subsection{Design of conjugated interferometer}

The CI is the key component of the gas sensing system. As shown in Figs. 1(a) and 1(b), the light source of the multi-component gas detection system consists of a broadband light source cascade CI filter. The CI includes two collimators and a sandwiched etalon. The etalon has two units: reference cell and absorption cell. The absorption cell is filled with the high concentration absorptive multi-gases for target gases wavelength, and the reference cell contains gas which is non-absorptive for target gases and its refractive index is closed to absorptive gas. We choose $\mathrm{NH}_{3}$ and $\mathrm{C}_{2} \mathrm{H}_{2}$ for garget gases in the paper.

The light from a broadband source is split into two parts by etalon and injected into two units. The amplitude powers can be expressed as

$$
\begin{gathered}
I_{1}(\lambda)=\sqrt{\eta_{\text {in }} r I(\lambda) \times[1-\alpha(\lambda)]} \\
I_{2}(\lambda)=\sqrt{\eta_{\text {in }}(1-r) I(\lambda)}
\end{gathered}
$$

where $I_{1}(\lambda)$ and $I_{2}(\lambda)$ are the amplitude powers of the absorption and reference units, $\eta_{\text {in }}$ is the insertion loss, $r$ is the splitting ratio of gas etalon, $I(\lambda)$ is the incident light intensity, and $\alpha(\lambda)$ is the gas absorption coefficient.

The output light intensity of the CI filter at the optical spectrum analyzer (OSA) is

$$
I_{0}(\lambda)=\eta_{\text {out }}\left[I_{1}^{2}+I_{2}^{2}+2 I_{1}(\lambda) I_{2}(\lambda) \text { co } \Delta \varphi\right]
$$

where $\Delta \varphi$ is the phase difference between the two 
units, and $\eta_{\text {out }}$ is the coupling coefficient from the

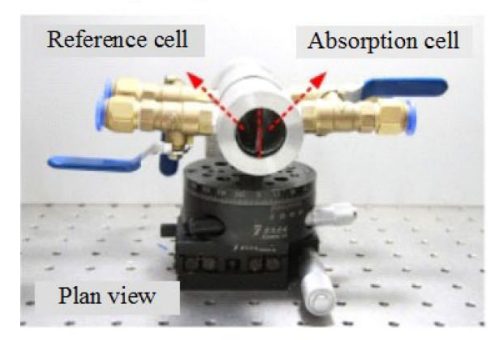

(a) etalon to the other fiber coupled collimator.

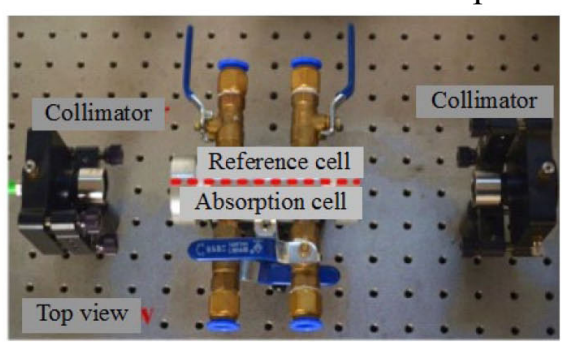

(b)

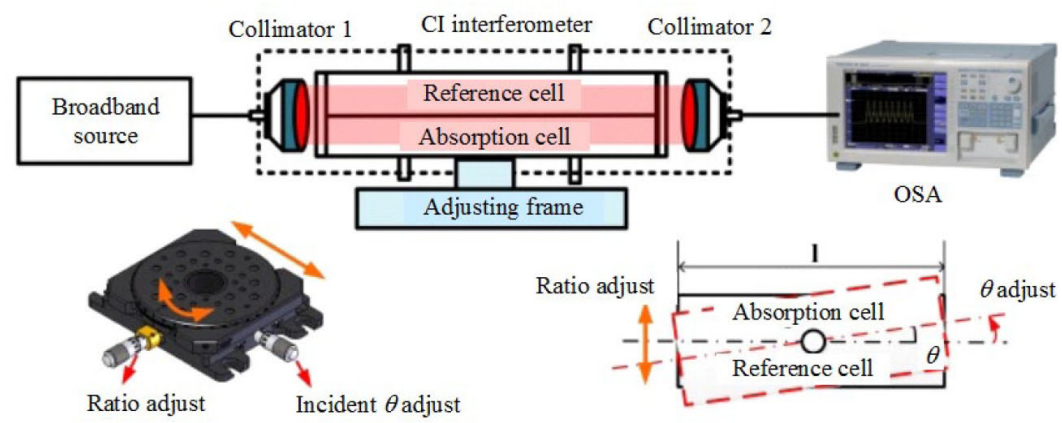

(c)

Fig. 1 Gas conjugated interferometer: (a) plan view of $\mathrm{CI}$, (b) top view of $\mathrm{CI}$, and (c) working principle of $\mathrm{CI}$.

If $r=50 \%$, (3) can be simplified as follows:

$I_{0}(\lambda)=\eta_{\text {in }} \eta_{\text {out }}\left[1-\frac{1}{2} \alpha(\lambda)+[1-\alpha(\lambda)] \cos \Delta \varphi\right] I(\lambda)$.

From (4), we can find that the transmission light intensity $I_{0}$ depends on the gas absorption coefficient $\alpha$ and the phase $\varphi$. When the phase different $\varphi=\pi$, (4) becomes

$$
I_{0}(\lambda)=\frac{1}{2} \alpha(\lambda) \eta_{\text {in }} \eta_{\text {out }} I(\lambda) \text {. }
$$

The important role of the phase difference can be expressed as

$$
\Delta \varphi=\frac{2 \pi}{\lambda}(\Delta n L / \cos \theta)
$$

where $\Delta n$ is the refractive index difference of the gases filled in two air cells, and $\theta$ is the incident angle. As shown in Fig. 1(c), the optical path difference $\Delta L$ in two gas cells is resulted from different refractive indexes and the shift of the rotation angle $\theta$. Different values of $\Delta n$ correspond to different output values of $\Delta L$, which also results in a change in $\varphi$ accordingly. In this essay, the $\pi$ phase shift condition can be achieved by altering the optical path difference (OPD) of the reference cell and absorption cell $(\Delta L)$. From (6), we can find that when $\Delta L=(\lambda / 2) \cdot(2 n+1),(n=0,1,2, \cdots)$, the $\pi$ phase shift condition is achieved. In order to make the absorption peak of the measuring spectral range flip at the same time, we must make the interference period greater, $\Delta L$ smaller, and the refractive indexes of two air cells as close as possible. Our targets gases are $\mathrm{C}_{2} \mathrm{H}_{2}$ and $\mathrm{NH}_{3}$, whose gas refractive indexes are 1.00051 and 1.000376 . Then we choose $\mathrm{CH}_{4}$ with a refractive index of 1.000444 , which has the most similar refractive index but at the same time whose absorption peak would not overlap with the target gas.

\subsection{Achievement of multi-component optical output}

The performance of the conjugated interferometer depends on the $\Delta \varphi, \alpha$, and the splitting ratio. We simulate and compare their effects around two assumed gas absorption lines at $1529 \mathrm{~nm}$ and $1531 \mathrm{~nm}$. The results are shown in Fig. 2. In the design and adjustment of the CI, controlling the 
parameter $\Delta \varphi$ to interval $(0.9 \pi, 1.1 \pi)$ and the

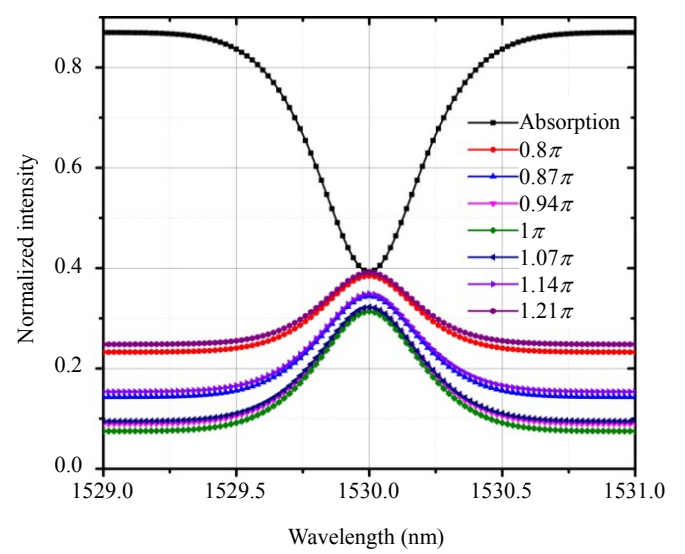

(a) splitting ratio to $(0.3,0.5)$ is better.

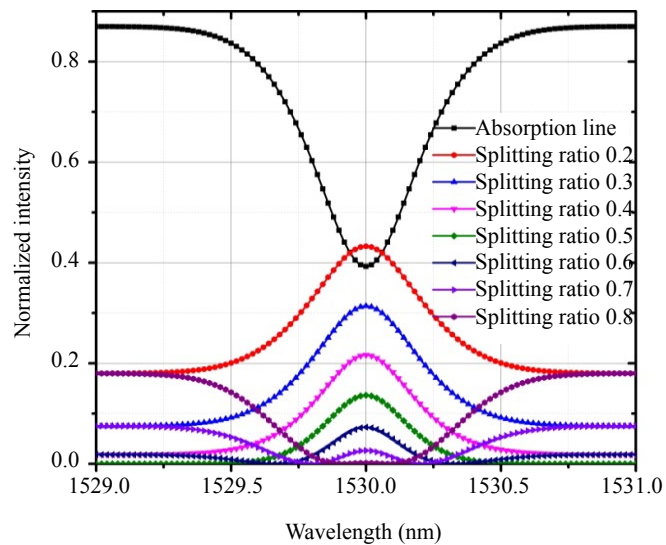

(b)

Fig. 2 Simulation transmission spectra of CI: (a) phase difference and (b) splitting ratio changes.

In a gas CI, we can simultaneously filter multi-component gases, so that among the outputs of the light source, we can obtain various light outputs of multi-component corresponding to gas absorption lines. As shown in Fig. 3, we use acetylene $\left(\mathrm{C}_{2} \mathrm{H}_{2}\right)$ and ammonia $\left(\mathrm{NH}_{3}\right)$ as absorptive gases, which have absorptions in the wavelength range of $1510 \mathrm{~nm}-$ $1535 \mathrm{~nm}$ and methane $\left(\mathrm{CH}_{4}\right)$ as the reference gas which has no absorption in the range of $1510 \mathrm{~nm}-$ $1535 \mathrm{~nm}$. By adjusting the incident $\theta$ to change the OPD, the $\pi$ phase shift condition is achieved. The

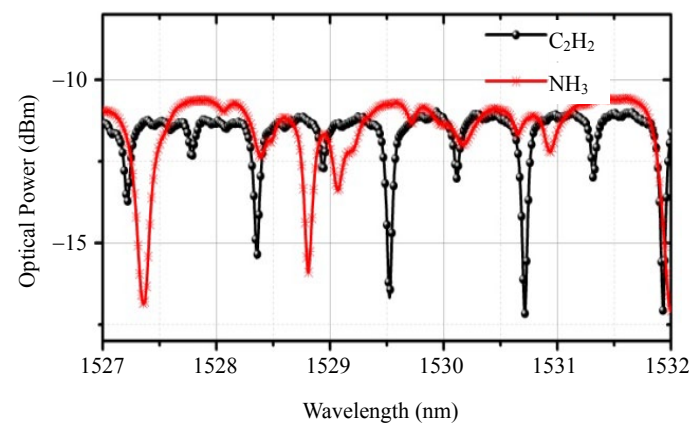

(a)

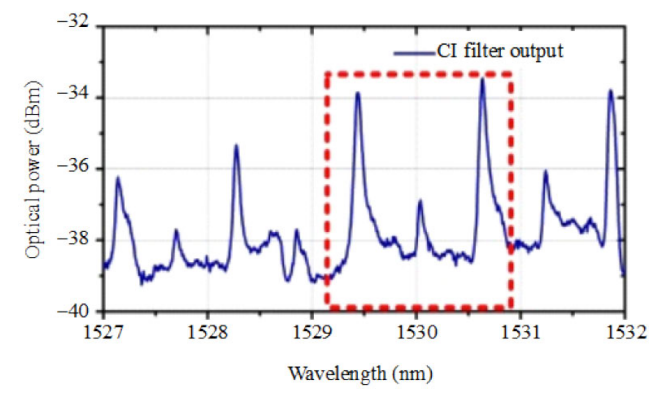

(c) gas absorption curve of the target gas is obtained by collecting the spectrum through the OSA. Figure 3 shows the transmission of the CI, and Figs.3(a) and 3(b) show two target gases absorption peaks in the range of $1527 \mathrm{~nm}-1532 \mathrm{~nm}$ and $1511 \mathrm{~nm}$ $1516 \mathrm{~nm}$. In these ranges, two target gases have some strong absorption peaks and do not affect each other. Figures 3(c) and 3(d) show the wavelength peaks of the CI match well with two target gases absorption lines throughout the wavelength ranges of $1527 \mathrm{~nm}-1532 \mathrm{~nm}$ and $1511 \mathrm{~nm}-1516 \mathrm{~nm}$.

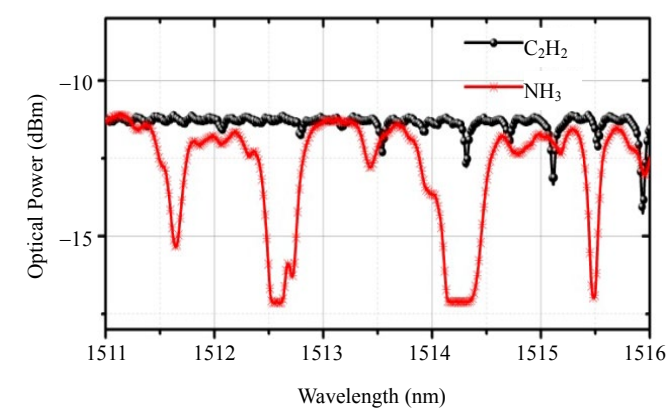

(b)

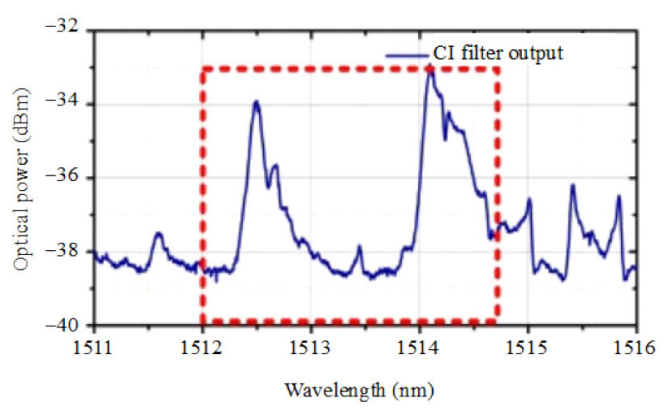

(d)

Fig. 3 Analysis of the CI filter: (a) absorption of $\mathrm{C}_{2} \mathrm{H}_{2}$ and $\mathrm{NH}_{3}(1527 \mathrm{~nm}-1532 \mathrm{~nm})$, (b) absorption of $\mathrm{C}_{2} \mathrm{H}_{2}$ and $\mathrm{NH}_{3}(1512 \mathrm{~nm}-$ $1516 \mathrm{~nm}),(\mathrm{c})$ CI filter output $(1527 \mathrm{~nm}-1532 \mathrm{~nm})$, and (d) CI filter output $(1512 \mathrm{~nm}-1516 \mathrm{~nm})$. 
Figure 4(a) shows the CI transmission spectrum range of $1510 \mathrm{~nm}-1535 \mathrm{~nm}$. The wavelength peaks of the CI match well with the gas absorption lines at the wavelength of $1510 \mathrm{~nm}-1535 \mathrm{~nm}$ from Hitran database [15]. Only light close to the gas absorption lines can effectively pass the CI. The background

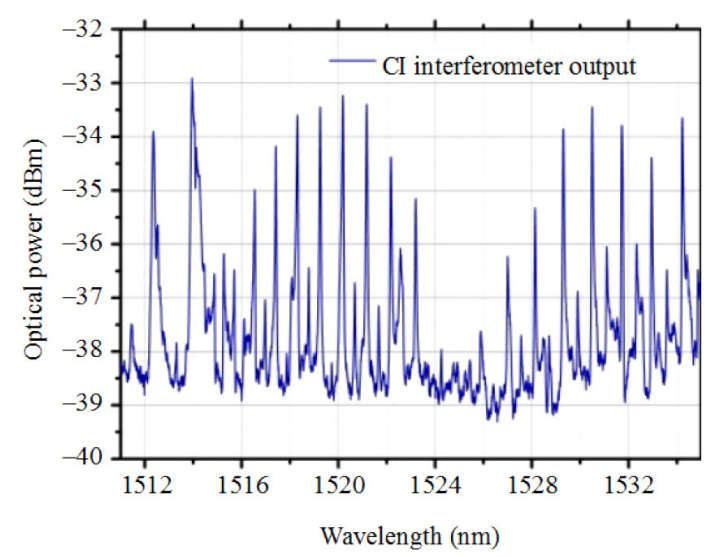

(a) light, which usually limits the gas detection sensitivity, is minimized with our CI. After that, we test the stability of the $\mathrm{CI}$ at room temperature and record the output spectrum of the CI 2 hours by every 10 min. As shown in Fig. 4(b), the CI has a good stability.

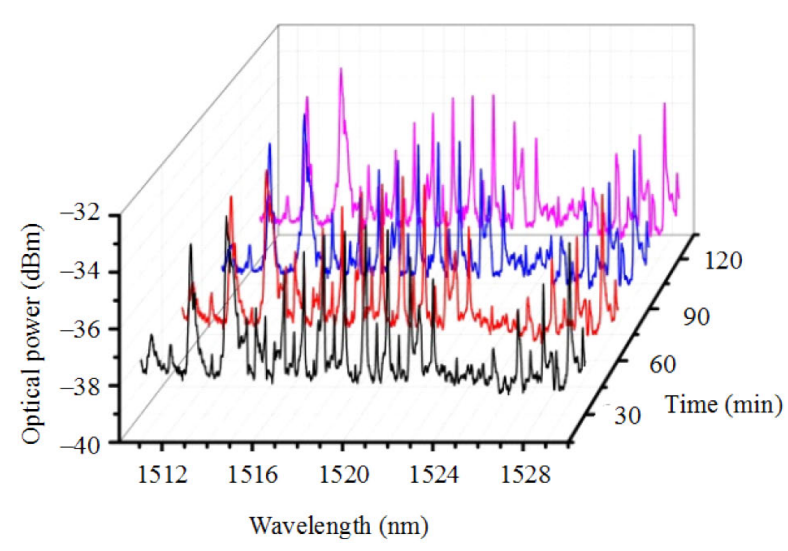

(b)

Fig. 4 Gas conjugated interferometer: (a) spectrum of CI and (b) stability of conjugated interferometer.

\section{Optical gas sensor system for multi-component gas}

We used $\mathrm{C}_{2} \mathrm{H}_{2}$ and $\mathrm{NH}_{3}$ gases as sample gases to test the multi-component gas concentration detection system. The multi-gas detection system was analyzed by qualitative and quantitative analysis.

\subsection{Multi-gases qualitative experiment}

The gas cell with a long-length of $1 \mathrm{~m}$ was connected to the CI filter to verify the feasibility of gas concentration detection. The results are shown in Fig. 5, when the different concentrations of $\mathrm{NH}_{3}$ and $\mathrm{C}_{2} \mathrm{H}_{2}$, respectively were filled in the gas cell, using the OSA on the spectrum acquisition. Due to the fact that the CI output contained the spectrum absorption information of $\mathrm{C}_{2} \mathrm{H}_{2}$ and $\mathrm{NH}_{3}$, when there was only one type of gas in the testing gas cell, for instance, under the condition that one of the testing gases was $\mathrm{C}_{2} \mathrm{H}_{2}$ gas, the output would be acetylene gas concentration information. When the optical signal contained the $\mathrm{C}_{2} \mathrm{H}_{2}$ gas concentration subtracting the initial signal, the insertion loss of testing gas was negligible, since the $\mathrm{NH}_{3}$ concentration did not change, the acquired signal would contain the $\mathrm{C}_{2} \mathrm{H}_{2}$ concentration information only, but the $\mathrm{NH}_{3}$ absorption spectrum information would be eliminated.

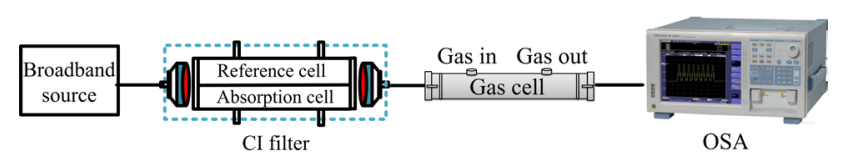

Fig. 5 Schematic of multi-gas basis verification test.

The two gases were analyzed by the above mentioned treatment method. Respectively, the $1000 \mathrm{ppm}$ and $2000 \mathrm{ppm}$ concentrations of $\mathrm{C}_{2} \mathrm{H}_{2}$, and the $0.5 \%$ and $1 \%$ concentrations of $\mathrm{NH}_{3}$ were filled into the detected gas cell. The four signals were collected from the OSA subtracting the initial signal, respectively. The results obtained are shown in Figs. 6 and 7. The concentration information of the corresponding gas matches well with the absorption spectrum information, and with an increase in the concentration, the absorption is stronger, which is consistent with the theory deduction. 


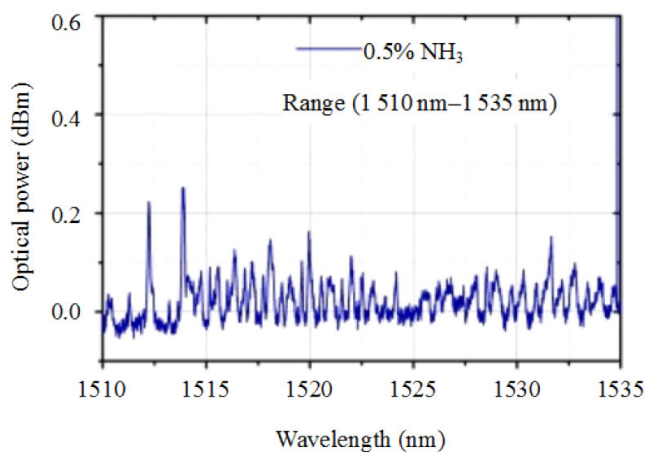

(a)

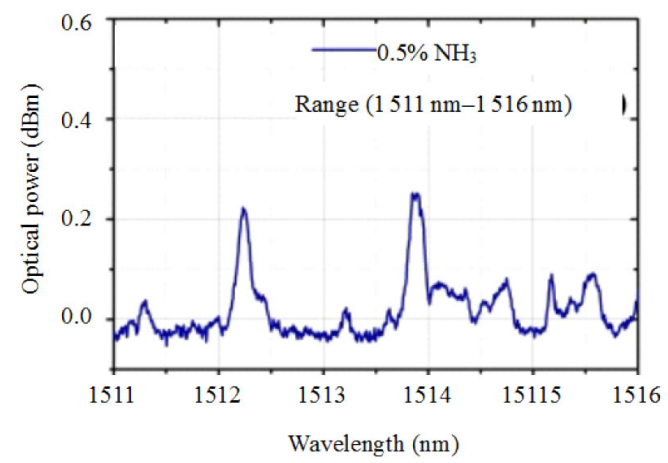

(c)

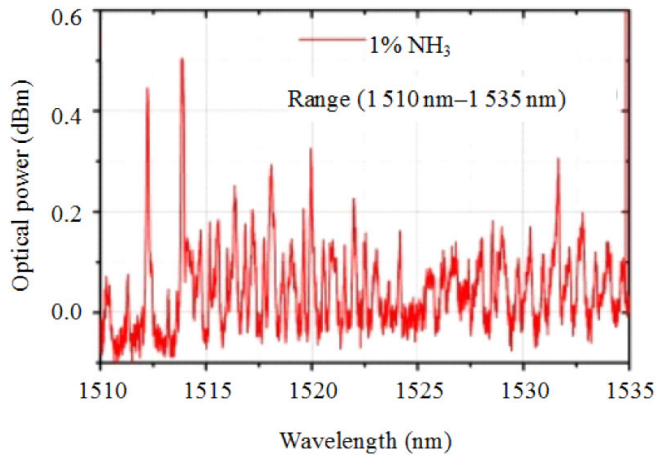

(b)

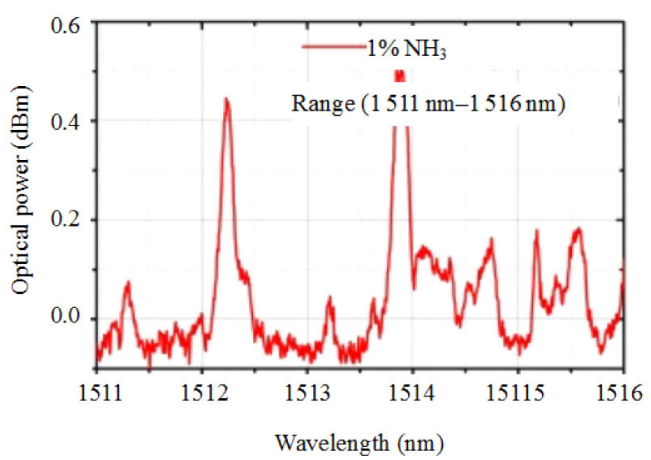

(d)

Fig. 6 Comparison of $\mathrm{NH}_{3}$ of different concentrations after data process: (a) $0.5 \% \mathrm{NH}_{3}\left(1510 \mathrm{~nm}-1535 \mathrm{~nm}\right.$ ), (b) $1 \% \mathrm{NH}_{3}(1510 \mathrm{~nm}$ $-1535 \mathrm{~nm})$, (c) $0.5 \% \mathrm{NH}_{3}(1511 \mathrm{~nm}-1516 \mathrm{~nm})$, and (d) $1 \% \mathrm{NH}_{3}(1511 \mathrm{~nm}-1516 \mathrm{~nm})$.

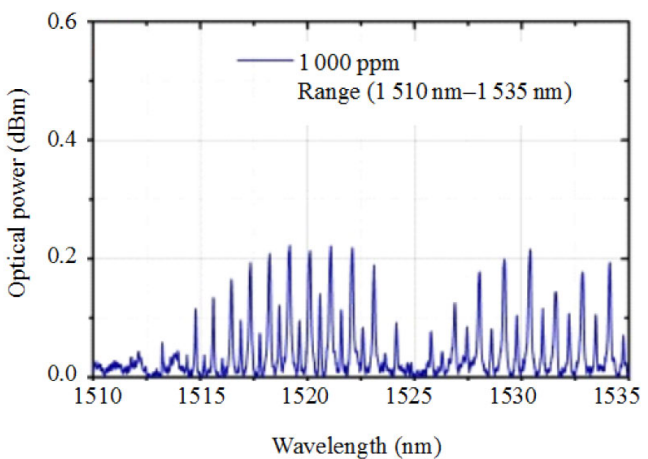

(a)

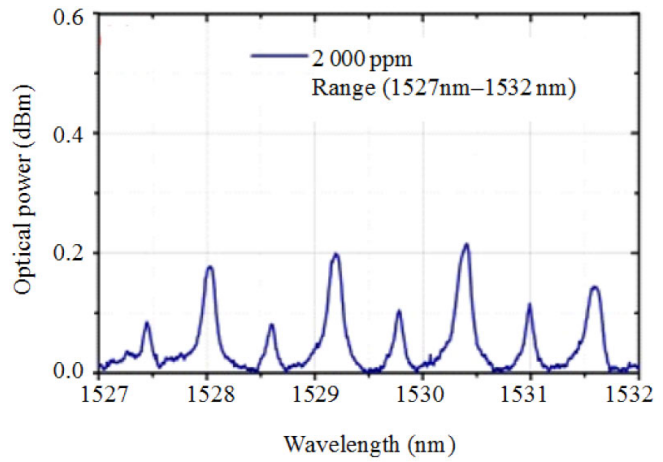

(c)

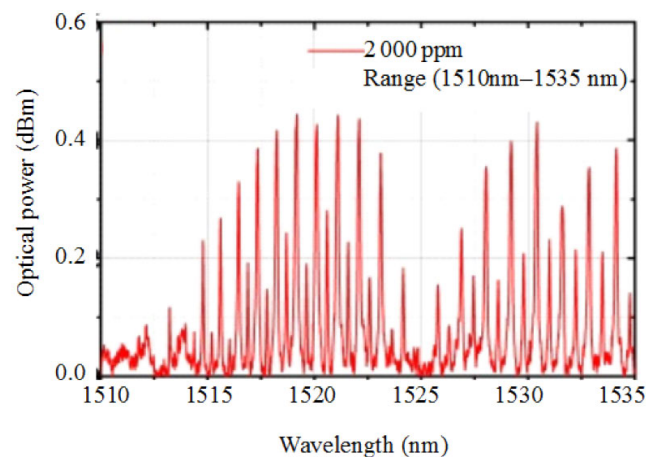

(b)

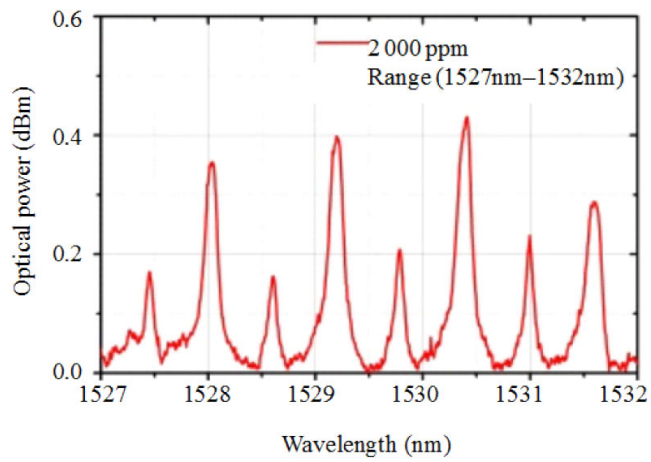

(d)

Fig. 7 Comparison of $\mathrm{C}_{2} \mathrm{H}_{2}$ of different concentrations after data process: (a) $1000 \mathrm{ppm} \mathrm{C}_{2} \mathrm{H}_{2}(1510 \mathrm{~nm}-1535 \mathrm{~nm})$, (b) $2000 \mathrm{ppm}$

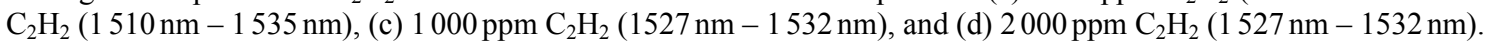




\subsection{Multi-gas experiment}

The schematic setup of the sensor prototype and its testing system are shown in Fig. 8. The system selected a broadband light source with a wavelength range of $1510 \mathrm{~nm}-1540 \mathrm{~nm}$ and a 1-m-length detection gas cell, and the optic propagation loss in the entire cell was less than $0.5 \mathrm{~dB}$. The light output of the CI was divided into two beams by a $90: 10$ coupler. The light beam with $10 \%$ power was detected by photo detector 1 (PD1) and served as a reference to compensate the source power fluctuation. Another light beam with $90 \%$ power passed the detection gas cell and an isolator, and then got detected by PD2. Data were recorded with a 16-bit data acquisition card.

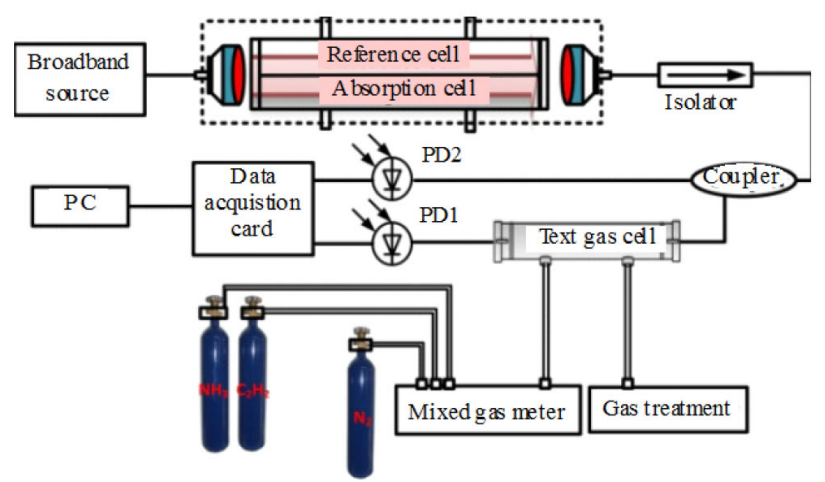

Fig. 8 Schematic of multiple gases detection system.

A reference channel was used to compensate for changes in the emission of the source, which were assumed to affect the reference and test channel wavelengths in the equal proportion. This relationship tended to result from the influence of the optoelectronic devices (such as coupler and PD). Thus, before the experiment, we should set a zero value calibration in the system to maintain that the concentration of the target gas was 0 (filled with pure $\mathrm{N}_{2}$ ) in the test cell, so there was a fixed linear relationship between the two signals collected. The experiment was carried out by introducing the gas of different concentrations and collecting light intensities of the two signals. Then we processed the light intensity by the linear relationship obtained by the calibration value of zero, and we could obtain complete gas concentration information after doing the difference.

A mixture of $\mathrm{NH}_{3}, \mathrm{C}_{2} \mathrm{H}_{2}$, and $\mathrm{N}_{2} \quad(99.99 \%$ concentration) was tested as the sample. The $\mathrm{NH}_{3}$ concentration was altered at $0.1 \%$ step in the range of $0 \%-1 \%$ by a mixing system (LFIX-2000), and the $\mathrm{C}_{2} \mathrm{H}_{2}$ concentration was altered at $200 \mathrm{ppm}$ step in the range of $0 \mathrm{ppm}-2000 \mathrm{ppm}$. For each step change in the concentration applied, a total of $2 \mathrm{~min}$ was allowed for passage of gas down the connecting pipe work, diffusion into the cell. At each concentration step, signals from the test and reference channels were recorded by data acquisition and the data were processed by the method mentioned above. The results are shown in Fig. 9. There were 10 concentrations of two target gases spectra information. Figure 9 reflects the result of subtraction between two channels in various gas concentrations. The main different fluctuations mainly concentrated on the range $(1511 \mathrm{~nm}-1516$ $\mathrm{nm})$ of $\mathrm{C}_{2} \mathrm{H}_{2}$ and the range $(1527 \mathrm{~nm}-1532 \mathrm{~nm})$ of $\mathrm{NH}_{3}$ absorption spectra, but no fluctuations in other wavelength ranges. The result could be determined accurately to reflect the concentration of the gas.

The ranges of the gas absorption peaks of $1511 \mathrm{~nm}-1516 \mathrm{~nm}$ and $1527 \mathrm{~nm}-1532 \mathrm{~nm}$ were selected corresponding to $\mathrm{NH}_{3}$ gas and $\mathrm{C}_{2} \mathrm{H}_{2}$ gas, respectively, and the wavelength range was integrated in order to eliminate subtraction errors caused by a low concentration. The integral value was the change in the light intensity information corresponding to the gas concentration. According to the Lambert-Beer law, the concentrations of the $\mathrm{C}_{2} \mathrm{H}_{2}$ and $\mathrm{NH}_{3}$ are in a linear relationship with the absorbance change $\Delta I$. The calibration curve is shown in Fig. 10. Figure 10(a) is a linear fit chart with a scale from $200 \mathrm{ppm}$ to $2000 \mathrm{ppm} \mathrm{C}_{2} \mathrm{H}_{2}$, and Fig. $10(\mathrm{~b})$ is a linear fit chart with a scale from $0.2 \%$ to $1 \% \mathrm{NH}_{3}$. The figure shows a good linearity of the two curves, $R^{2}=0.9987$ and $R^{2}=0.9968$. The lowest detections of the system are $20 \mathrm{ppm}$ of $\mathrm{C}_{2} \mathrm{H}_{2}$ and $0.01 \% \mathrm{NH}_{3}$. 


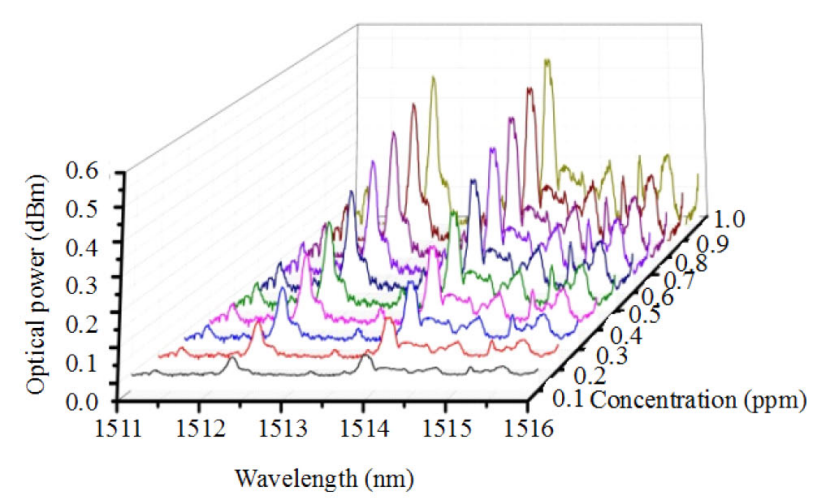

(a)

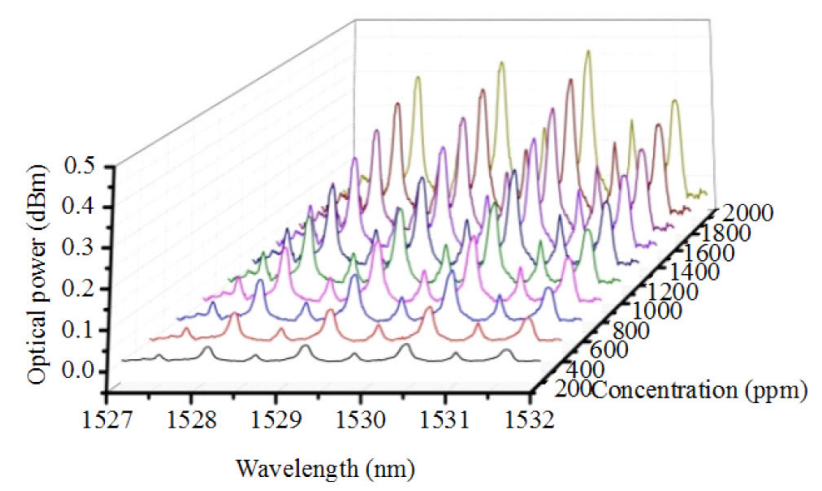

(b)

Fig. 9 Data process of the gas concentration test: (a) $\mathrm{NH}_{3}$ and (b) $\mathrm{C}_{2} \mathrm{H}_{2}$.

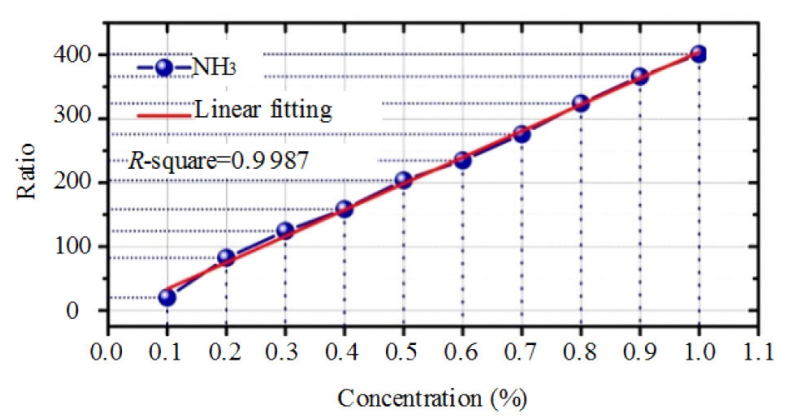

(a)

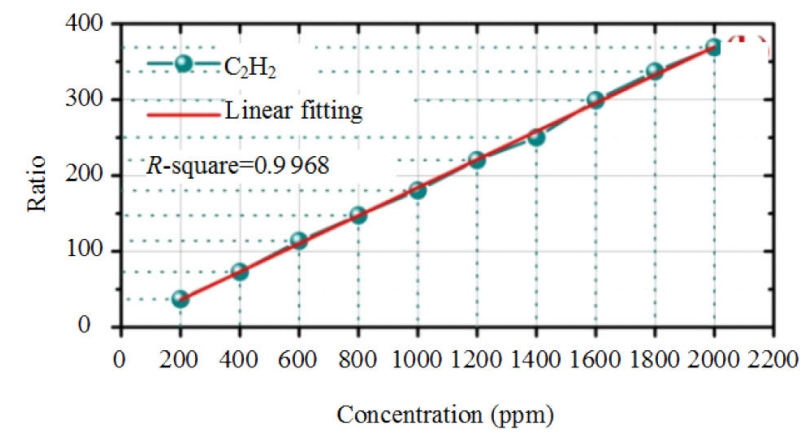

(b)

Fig. 10 Gas detection system concentration analysis: (a) $\mathrm{NH}_{3}$ and (b) $\mathrm{C}_{2} \mathrm{H}_{2}$.

\section{Conclusions}

In this program, an optical multi-component gas sensor detection system based on the conjugated interferometer has been set up. The CI can transform the absorption spectrum of the target gases to a conjugated emission spectrum, and when combining the CI with the broadband light source, the spectrum of output light matches well with the absorption spectrum of target gases. The CI design for different target gases can be achieved by replacing the kind of target absorbing gases in the CI filter. Concerning the multi-component gas detection, the differential detection system based on the CI developed in this paper can be applied in the detection of multi-component gas detection, and the inversion of gas concentration with the output result of the system can be highly reliable as well. According to the experimental results, the system can be applicable in the multi-component gas concentration detection under the coexistence of various gases. The lowest detection of system is $20 \mathrm{ppm}$ of $\mathrm{C}_{2} \mathrm{H}_{2}$ and $0.01 \% \mathrm{NH}_{3}$.

\section{Acknowledgment}

This research was supported by the Natural National Science Foundation of China, NSFC (Grant No. 61575149, 61290311), and the Major Project of Hubei Technological Innovation Special Fund (2016AAA008).

Open Access This article is distributed under the terms of the Creative Commons Attribution 4.0 International License (http://creativecommons.org/ licenses/by/4.0/), which permits unrestricted use, distribution, and reproduction in any medium, provided you give appropriate credit to the original author(s) and the source, provide a link to the Creative Commons license, and indicate if changes were made.

\section{References}

[1] R. Dhawan, M. M. Khan, N. Panwar, U. Tiwari, R. Bhatnagar, S. C. Jain, et al., "A low loss mechanical splice for gas sensing using hollow-core photonic crystal fibre," Optik - International Journal for Light 
and Electron Optics, 2013, 124(18): 3671-3673.

[2] S. Schilt, L. Thevenaz, M. Nikles, L. Emmenegger, and C. Huglin, "Ammonia monitoring at trace level using photoacoustic spectroscopy in industrial and environmental applications," Spectrochimica Acta Part A Molecular \& Biomolecular Spectroscopy, 2004, 60(14): 3259-3268.

[3] D. Smith and P. Spanel, "The challenge of breath analysis for clinical diagnosis and therapeutic monitoring," Analyst, 2007, 132(5): 390-396.

[4] R. J. Lu, D. M. Shen, Q. Q. Du, B. Z. Huang, and J. S. Shi, "Tuning characteristics of DFB diode laser and its application to TDLAS gas sensor design," Applied Mechanics \& Materials, 2014, 511: 173-177.

[5] M. Jahjah, R. Lewicki, K. F. Tittle, K. Krzempek, P. Stefanski, S. So, et al., "CW DFB RT diode laser-based sensor for trace-gas detection of ethane using a novel compact multi-pass gas absorption cell," SPIE, 2013, 112(4): 461-465.

[6] J. Ye and Z. A. Li, "Method for the measurement of methane gas based on multi-beam interferometry," Journal of the Optical Society of Korea, 2013, 17(6): 481-485.

[7] H. Ding, J. Q. Liang, J. H. Cui, and X. N. Wu, "A novel fiber Fabry-Perot filter based mixed-gas sensing system," Sensors \& Actuators B: Chemical, 2009, 138(1): 154-159.

[8] K. L. Chan, Z. Ning, D. Westerdahl, K. C .Wong, Y. W. Sun, A. Hartl, et al., "Dispersive infrared spectroscopy measurements of atmospheric $\mathrm{CO}_{2}$ using a Fabry-Perot interferometer sensor," Science of the
Total Environment, 2014, 472: 27-35.

[9] W. Jin, S. Murray, D. Pinchbeck, G. Stewart, and B. Culshaw, "Absorption measurement of methane gas with a broadband light source and interferometric signal processing," Optics Letters, 1993, 18(16): 1364.

[10] J. Hodgkinson, R. Smith, W. O. Ho, J. R. Saffell, and R. P. Tatam, "Non-dispersive infra-red (NDIR) measurement of carbon dioxide at $4.2 \mu \mathrm{m}$ in a compact and optically efficient sensor," Sensors \& Actuators B: Chemical, 2013, 186(186): 580-588.

[11] Z. P. Zhu, Y. H. Xu, and B. G. Jiang, "A one ppm NDIR methane gas sensor with single frequency filter denoising algorithm," Sensors, 2012, 12(9): 12729-12740.

[12] F. Bilodeau, K. O. Hill, B. Malo, D. C. Johnson, and J. Albert, "High-return-loss narrowband all-fiber bandpass Bragg transmission filter," IEEE Photonics Technology Letters, 1994, 6(1): 80-82.

[13] P. Q. Liu, X. Wang, and C. F. Gmachl, "Single-mode quantum cascade lasers employing asymmetric Mach-Zehnder interferometer type cavities" Applied Physics Letters, 2012, 101(16): 219-221.

[14] Z. Y. Li, X. Gui, C. C. Hu, L. Zheng, H. H. Wang, and J. M. Gong, "Optical gas sensor based on gas conjugated interference light source," IEEE Photonics Technology Letters, 2015, 27(14): 1550-1552.

[15] HITRAN on the Web. Available online: http://hitran. iao.ru (accessed on 1 July 2012). 